\title{
WANNIER FUNCTIONS FOR LATTICES IN A MAGNETIC FIELD II: EXTENSION TO IRRATIONAL FIELDS
}

\author{
Michael Wilkinson* \\ Department of Physics and Applied Physics, \\ John Anderson Building, \\ University of Strathclyde, \\ Glasgow, G4 0NG, \\ Scotland, U.K.
}

\begin{abstract}
This paper extends earlier work on the definition of Wannier functions for Bloch electrons in a magnetic field. Extensions to irrational as well as rational magnetic fields are defined, and their properties investigated. The results are used to give a generalisation of the Peierls effective Hamiltonian which is valid when the magnetic flux per unit cell is close to any rational number.
\end{abstract}

\footnotetext{
* Address after 1st August 2000: Faculty of Mathematics and Computing, The Open University, Milton Keynes, MK7 6AA, Bucks, England.
} 


\section{Introduction}

Wannier functions are localised basis states which span a band of Bloch eigenfunctions [1]. The use of localised basis functions can be convenient both technically and conceptually, particularly when considering perturbations which are themselves spatially localised. There are difficulties in defining satisfactory Wannier states when a magnetic field is applied to the lattice. Firstly, the eigenfunctions are typically not Bloch states: in two dimensions the eigenfunctions are only Bloch states if the ratio $\beta$ of the flux quantum to the magnetic flux per unit cell is a rational number $[2,3]$ (in these cases I will write $\beta=p / q$, where $p$ and $q$ are integers with no common divisor). Secondly, even when the magnetic field is rational in this sense, conventional Wannier states only have satisfactory localisation properties if a topological invariant (the Chern index) chararterising a Bloch band is equal to zero $[4,5]$. In a previous paper (reference [6]) it was shown how this latter difficulty could be overcome, for two dimensional lattices, in the case where the magentic flux per unit cell is rational. In [6] I showed how to obtain a complete set of states which span a Bloch band, and which retain all of the useful properties of conventional Wannier functions. Two different definitions were examined, termed type I and type II Wannier functions. The definition of these states contains the Chern index $M$ of the band, and in both cases they reduce to the conventional Wannier function when $M=0$.

The purpose of the present paper is twofold. The first objective is to show how the definition of Wannier functions can be usefully extended to irrational fields, despite the fact that Bloch bands do not exist in this case. Some of the results for type II Wannier functions are anticipated in earlier papers by the same author [7], [8] (the latter in collaboration with R. J. Kay). These earlier papers discussed 'irrational' generalised Wannier functions for the special case of the 'phase space lattice Hamiltonian', a one dimensional model which reprenents many of the features of Bloch electrons in a magnetic field. The form of the Wannier functions of the full Hamiltonian for irrational magnetic fields is related to the results for the phase space lattice Hamiltonian, but the generalisations are not obvious. The derivation given here is also more satisfying in that it uses only minimal algebraic properties, and that results are obtained for both types of Wannier functions introduced in $[6]$.

The second objective is to use the generalised Wannier functions to obtain a very general form of the Peierls effective Hamiltonian $[9,10]$, in a form suitable for systematic analysis. For simplicity we consider only a two-dimensional case where the electron is confined to a plane, and perturbed by a magnetic field in the perpendicular direction (with cartesian coordinate $z$ ). A comprehensive treatment of the three dimensional case introduces the complication of an additional commensurability parameter, but is straightforward when the field is aligned with one of the crystal axes. The Peierls Hamiltonian is a one-dimensional effective Hamiltonian which describes the effect of a uniform magnetic field perturbing a band of Bloch states. If the dispersion relation is $\mathcal{E}\left(k_{x}, k_{y}\right)$, the Peierls effective Hamiltonian takes the form

$$
\hat{H} \sim \mathcal{E}\left(\hat{K}_{x}, \hat{K}_{y}\right)
$$

where $\hat{K}_{x}$ and $\hat{K}_{y}$ are generators of the magnetic translation operators, $\hat{T}(\mathbf{R})$ (These are defined in section 3 ; they were introduced in [11,12], and are discussed concisely in [6]). These satisfy

$$
\left[\hat{K}_{x}, \hat{K}_{y}\right]=\mathrm{i} \frac{2 \pi \beta^{-1}}{\left|\mathbf{A}_{1} \wedge \mathbf{A}_{2}\right|}
$$

where $\mathbf{A}_{i}$ are the basis vectors for the lattice. Many derivations of this relationship exist where the dispersion relation is that of the $B=0$ problem. This paper considers the case 
where the dispersion relation is that of the system with any rational magnetic field $p / q$, showing that the Peierls effective Hamiltonian is applicable in this case. It is shown that the commutator (1.2) is replaced by one which depends upon the Chern index: the general form of $(1.2)$ is

$$
\left[\hat{K}_{x}, \hat{K}_{y}\right]=\mathrm{i} \frac{2 \pi \gamma}{\left|\mathbf{A}_{1} \wedge \mathbf{A}_{2}\right|}
$$

where $\gamma$ is another dimensionsionless parameter characterising the magnetic field. The value of $\gamma$ depends upon the value of the Chern integer $M$, and upon another integer $N$ which satisfies

$$
q M+p N=1 .
$$

The dimensionless effective magnetic field $\gamma$ is

$$
\gamma=\frac{q \beta-p}{M+N \beta}
$$

The expression (1.5) can be surmised from results obtained previously for the phase space lattice Hamiltonian [7,8]. The derivation presented here indicates how the effective Hamiltonian can be obtained for the full Hamiltonian, rather than a one-dimensional model. This issue has also been considered by Chang and Niu [13], who also discussed an heuristic approach to determining the first order correction to the effective Hamiltonian. The method described here allows a systematic development of the effective Hamiltonian, using similar techniques to those applied to the phase space lattice Hamiltonian in reference [14]. It also has the advantage that some of the complicated intermediate steps in the algebra of references [7] and [14] are given a more transparent interpretation.

Sections 2 and 3 respectively summarise the essential definitions and principal results from [6], and a representation of the Hamiltonian as a sum of magnetic translation operators. The latter will be essential to the derivation of the general effective Hamiltonian.

Section 4 describes the extension of the Wannier functions obtained in [6] to irrational magnetic fields, and a corresponding extension of the definition of Bloch states. The next four sections consider various properties of the generalised Wannier functions and Bloch states. Section 5 discusses the effect upon the Wannier functions of a transformation of the Bloch states. A 'gauge transformation' of the form

$$
|B(\mathbf{k})\rangle \rightarrow\left|B^{\prime}(\mathbf{k})\right\rangle=\exp [\mathrm{i} \theta(\mathbf{k})]|B(\mathbf{k})\rangle
$$

is applied to the Bloch states, with $\theta(\mathbf{k})$ a periodic function. The Wannier states derived from the gauge transformed Bloch states can be obtained from the original Bloch states by the action of an operator, which is obtained in section 5. Similarly, section 6 determines an operator acting on the Wannier states which is the image of a translation operator acting on the Bloch states.

Section 7 computes the Dirac bracket of two generalised Bloch states, $\left\langle B^{\prime}\left(\mathbf{k}^{\prime}\right) \mid B(\mathbf{k})\right\rangle$, which will be required for determining matrix elements of the Hamiltonian. Section 8 introduces some notational devices which simplify and illuminate the rather complex expressions obtained earlier, showing how they can written in terms of translation operators with algebra analogous to that of the magnetic translation group. Finally, in section 9 these results are used to obtain the general form for the Peierls efffective Hamiltonian. 


\section{Summary of earlier results}

The purpose of this section is to present, for the convenience of the reader, a summary of some of the principal definitions and equations from the earlier paper, reference [6]. The lattice vectors are written $\mathbf{R}=n_{1} \mathbf{A}_{1}+n_{2} \mathbf{A}_{2}$, and the reprocal lattice vectors are $\mathbf{K}=n_{1} \mathbf{a}_{1}+n_{2} \mathbf{a}_{2}$, with $\mathbf{a}_{i} \cdot \mathbf{A}_{j}=2 \pi \delta_{i j}$.

The magnetic translation operators $\hat{T}(\mathbf{R})$ introduced by Zak [11] and Brown [12] are of fundamental importance. They are a representation of the symmetry of the system: if $\mathbf{R}$ is a lattice vector, the $\hat{T}(\mathbf{R})$ commutes with the Hamiltonian. The magnetic translation operators do not commute among themselves, and their composition rule can be written in the form

$$
\hat{T}\left(\mathbf{R}_{1}\right) \hat{T}\left(\mathbf{R}_{2}\right)=\exp \left[\frac{\pi \mathrm{i}}{\beta} \frac{\left(\mathbf{R}_{1} \wedge \mathbf{R}_{2}\right)}{\left(\mathbf{A}_{1} \wedge \mathbf{A}_{2}\right)}\right] \hat{T}\left(\mathbf{R}_{1}+\mathbf{R}_{2}\right)
$$

where $\beta$ is the flux quantum divided by the magnetic flux per unit cell. The magnetic translation operators are discussed concisely in [6].

When conventional Wannier functions are defined, it is assumed that the Bloch states are periodic functions of the Bloch wavevector $\mathbf{k}$, as well as being eigenfunctions of the lattice translation operators $\hat{T}\left(\mathbf{A}_{i}\right)$, with eigenvalues $\exp \left[\mathbf{i k} . \mathbf{A}_{i}\right]$. In the case where a rational magnetic field (with $q / p$ flux quanta per unit cell) is applied, in general both of these conditions need to be modified. The Bloch states are $p$ fold degenerate, and their phase increases by $2 \pi M$ on traversing the boundary of the unit cell. Throughout this paper, the following choice for the eigenvalue and periodicity conditions is preferred:

$$
\begin{gathered}
\hat{T}\left(\mathbf{A}_{1}\right)|B(\mathbf{k})\rangle=\exp \left[\mathrm{ik} \cdot \mathbf{A}_{1}\right]\left|B\left(\mathbf{k}-q \mathbf{a}_{2} / p\right)\right\rangle \\
\hat{T}\left(\mathbf{A}_{2}\right)|B(\mathbf{k})\rangle=\exp \left[\mathrm{ik} \cdot \mathbf{A}_{2}\right]|B(\mathbf{k})\rangle \\
\left|B\left(\mathbf{k}+\mathbf{a}_{1} / p\right)\right\rangle=\exp \left[\mathrm{i} M \mathbf{k} \cdot \mathbf{A}_{2}\right]|B(\mathbf{k})\rangle \\
\left|B\left(\mathbf{k}+\mathbf{a}_{2}\right)\right\rangle=|B(\mathbf{k})\rangle .
\end{gathered}
$$

Bloch states with their phases chosen to satisfy (2.2a) and (2.2b), and with degenerate states resolved so that $(2.2 \mathrm{c})$ is satisfied will be termed canonical Bloch states. Except when $p=1$ and $M=0$, these conditions depend upon the choice of lattice basis vectors $\mathbf{A}_{i}$.

The method for constructing the Wannier functions is based upon the following observation: if the Bloch states are canonical, the state $|C(\mathbf{k})\rangle=\hat{T}\left(-p M \mathbf{k} . \mathbf{A}_{2} / 2 \pi\right)|B(\mathbf{k})\rangle$ is periodic on the Brillouin zone of the superlattice spanned by $p \mathbf{A}_{1}, \mathbf{A}_{2}$, and Wannier functions $|\chi(\mathbf{R})\rangle$ are obtained by integrating the state $|C(\mathbf{k})\rangle$ with weight $\exp [\mathbf{i k . R}]$. In the case of standard Wannier functions, all of the Wannier states are obtained be applying translation operators to a single fundamental Wannier state. In the magnetic case, the full set of Wannier states is obtained by applying lattice translations to $|N|$ fundamental type I Wannier states, $\left|\chi_{\mu}\right\rangle=\left|\chi\left(\mu \mathbf{A}_{1}\right)\right\rangle, \mu=0, . .,|N|-1$, where $N$ satisfies (1.4). The relation between the Bloch and type I Wannier states is

$$
\begin{gathered}
|B(\mathbf{k})\rangle=\sum_{\mathbf{R}=n_{1} \mathbf{A}_{1}+n_{2} \mathbf{A}_{2}} \exp [-\mathrm{ik} \cdot \mathbf{R}] \hat{T}\left(n_{2} \mathbf{A}_{2}\right) \hat{T}\left(n_{1} \mathbf{A}_{1}\right) \hat{T}\left(\frac{p M}{2 \pi}\left(\mathbf{k} \cdot \mathbf{A}_{1}\right) \mathbf{A}_{2}\right) \\
\times \sum_{\mu=0}^{|N|-1} \exp \left[-\mathrm{i} p \mu\left(\mathbf{k} \cdot \mathbf{A}_{1}\right)\right]\left|\chi_{\mu}\right\rangle .
\end{gathered}
$$


A somewhat more natural representation of the Bloch states uses an alternative set of fundamental Wannier states: the type II Wannier states are defined by

$$
\left|\phi_{\mu}\right\rangle=\frac{1}{N} \sum_{\mu^{\prime}=0}^{|N|-1} \exp \left[-2 \pi \mathrm{i} \mu \mu^{\prime} / N\right] \hat{T}\left(-\mu^{\prime} \mathbf{A}_{1} / N\right)\left|\chi_{\mu^{\prime}}\right\rangle .
$$

One advantage of using the type II Wannier states is that upon expanding the Bloch states in terms of the $\left|\phi_{\mu}\right\rangle$ states, the summation over $\mu$ no longer depends upon $\mathbf{k}$ : the Bloch states are given in terms of the type II states by the relation

$$
\begin{gathered}
|B(\mathbf{k})\rangle=\sum_{\mathbf{R}=n_{1} \mathbf{A}_{1} / N+n_{2} \mathbf{A}_{2}} \exp [-\mathrm{ik} \cdot \mathbf{R}] \sum_{\mu=0}^{|N|-1} \exp \left[2 \pi \mathrm{i} n_{1} \mu / N\right] \\
\times \hat{T}\left(n_{2} \mathbf{A}_{2}\right) \hat{T}\left(n_{1} \mathbf{A}_{1} / N\right) \hat{T}\left(\frac{p M}{2 \pi}\left(\mathbf{k} \cdot \mathbf{A}_{1}\right) \mathbf{A}_{2}\right)\left|\phi_{\mu}\right\rangle .
\end{gathered}
$$

The other advantage of the type II Wannier states is that their transformations under a change of lattice basis vectors are simpler [6].

\section{The Hamiltonian in terms of translation operators}

Here the objective is to represent the Hamiltonian as a sum of magnetic translation operators: this will facilitate the construction of the effective Hamiltonian. The Hamiltonian is

$$
\begin{gathered}
\hat{H}=\frac{1}{2 m}(\hat{\mathbf{p}}-e \mathbf{A}(\hat{\mathbf{r}}))^{2}+V(\hat{\mathbf{r}}) \\
V(\mathbf{r})=V(\mathbf{r}+\mathbf{R}), \quad \mathbf{R}=n_{1} \mathbf{A}_{1}+n_{2} \mathbf{A}_{2}
\end{gathered}
$$

with the magnetic field generated by a linear vector potential, constructed using a matrix $\tilde{\mathcal{B}}$ with elements $\mathcal{B}_{i j}$ :

$$
\mathbf{A}(\mathbf{r})=\tilde{\mathcal{B}} \mathbf{r}, \quad \nabla \wedge \mathbf{A}=B \mathbf{e}_{3}, \quad \mathcal{B}_{21}-\mathcal{B}_{12}=B
$$

The magnetic translations $\hat{T}(\mathbf{R})$ have a generator $\hat{\mathbf{P}}=\hat{P}_{1} \mathbf{e}_{1}+\hat{P}_{2} \mathbf{e}_{2}$ :

$$
\begin{aligned}
\hat{T}(\mathbf{R}) & =\exp [-\mathrm{i} \hat{\mathbf{P}} \cdot \mathbf{R} / \hbar] \\
\hat{\mathbf{P}} & =\hat{\mathbf{p}}-e \tilde{\mathcal{B}}^{T} \hat{\mathbf{r}}
\end{aligned}
$$

It will also be useful to define a set of conjugate generators $\hat{P}_{i}^{*}$ :

$$
\hat{\mathbf{P}}^{*}=\hat{\mathbf{p}}-e \tilde{\mathcal{B}} \hat{\mathbf{r}} .
$$

The generators $\hat{P}_{i}, \hat{P}_{i}^{*}$ satisfy the commutation relations, where $\varepsilon_{i j}$ is the antisymmetric symbol, with elements $\varepsilon_{11}=\varepsilon_{22}=0, \varepsilon_{12}=-\varepsilon_{21}=1$ :

$$
\begin{aligned}
& {\left[\hat{P}_{i}, \hat{P}_{j}\right]=-\mathrm{i} e \hbar B \varepsilon_{i j}} \\
& {\left[\hat{P}_{i}^{*}, \hat{P}_{j}^{*}\right]=\mathrm{i} e \hbar B \varepsilon_{i j}}
\end{aligned}
$$




$$
\left[\hat{P}_{i}, \hat{P}_{j} *\right]=0 .
$$

The coordinate vector $\hat{\mathbf{r}}$ can be expressed in terms of the generators $\hat{\mathbf{P}}$ and $\hat{\mathbf{P}}^{*}$ : from (3.4) and (3.5) it follows that $\hat{P}_{i}^{*}-\hat{P}_{i}=e B \varepsilon_{i j} \hat{r}_{j}$ (where, from here until the end of section 3 , repeated indices are summed over). This can be inverted to give

$$
\hat{r}_{i}=\frac{1}{e B} \varepsilon_{i j}\left(\hat{P}_{j}-\hat{P}_{j}^{*}\right) .
$$

The Hamiltonian can now be written

$$
\hat{H}=\frac{1}{2 m} \hat{P}_{i}^{*} \hat{P}_{i}^{*}+\sum_{\mathbf{k}} V_{\mathbf{k}} \exp [\mathbf{i k} \cdot \hat{\mathbf{r}}]
$$

where the $\mathbf{k}=n_{1} \mathbf{a}_{1}+n_{2} \mathbf{a}_{2}$ are vectors in the reciprocal lattice, with basis vectors satisfying $\mathbf{a}_{i} \cdot \mathbf{A}_{j}=2 \pi \delta_{i j}$. Expressing the $\hat{\mathbf{r}}$ using (3.9), and using the fact that $\hat{P}_{i}$ and $\hat{P}_{j}^{*}$ commute, (3.10) can be written in the form

$$
\begin{gathered}
\hat{H}=\frac{1}{2 m} \hat{P}_{i}^{*} \hat{P}_{i}^{*}+\sum_{\mathbf{k}} V_{\mathbf{k}} \exp \left[\mathrm{i} k_{i} \varepsilon_{i j} \hat{P}_{j} / e B\right] \exp \left[-\mathrm{i} k_{i} \varepsilon_{i j} \hat{P}_{j}^{*} / e B\right] \\
=\frac{1}{2 m} \hat{P}_{i}^{*} \hat{P}_{i}^{*}+\sum_{\mathbf{k}} V_{\mathbf{k}} \hat{T}^{*}\left(-\hbar \mathbf{k}^{*} / e B\right) \hat{T}\left(\hbar \mathbf{k}^{*} / e B\right)
\end{gathered}
$$

where $\mathbf{k}^{*}=k_{i}^{*} \mathbf{e}_{i}$ and $\hat{T}^{*}(\mathbf{R})$ are defined by

$$
k_{i}^{*}=\varepsilon_{i j} k_{j}, \quad \hat{T}^{*}(\mathbf{R})=\exp \left[-\mathrm{i} \hat{\mathbf{P}}^{*} \cdot \mathbf{R} / \hbar\right] .
$$

The Hamiltonian is therefore expressed in terms of a sum of magnetic translation operators, with operator-valued coefficients $\hat{V}_{\mathbf{k}}$ :

$$
\hat{H}=\sum_{\mathbf{k}=n_{1} \mathbf{a}_{1}+n_{2} \mathbf{a}_{2}} \hat{V}_{\mathbf{k}} \hat{T}\left(\hbar \mathbf{k}^{*} / e B\right) .
$$

The operators $\hat{V}_{\mathbf{k}}$ commute with the magnetic translation operators, and are given by

$$
\hat{V}_{\mathbf{k}}=\frac{1}{2 m} \delta_{\mathbf{k}, \mathbf{0}} \hat{\mathbf{P}}^{*} \cdot \hat{\mathbf{P}}^{*}+V_{\mathbf{k}} \hat{T}^{*}\left(-\hbar \mathbf{k}^{*} / e B\right) .
$$

It is desireable to express the vectors $\mathbf{k}^{*}$ in terms of the real-space lattice basis vectors $\mathbf{A}_{1}$, $\mathbf{A}_{2}$. The vectors corresponding to reciprocal lattice vectors $\mathbf{a}_{i}$ are denoted by $\mathbf{k}_{i}^{*}$. Writing $\mathbf{A}_{i}=A_{i j} \mathbf{e}_{j}$ and $\mathbf{a}_{i}=a_{i j} \mathbf{e}_{j}$, the matrices $\tilde{A}=\left\{A_{i j}\right\}$ and $\tilde{a}=\left\{a_{i j}\right\}$ satisfy $\tilde{A} \tilde{a}^{T}=2 \pi \tilde{I}$. It follows that

$$
\begin{aligned}
& \mathbf{k}_{1}^{*}=\frac{2 \pi}{\operatorname{det}(\tilde{A})}\left(-A_{21} \mathbf{e}_{1}-A_{22} \mathbf{e}_{2}\right) \\
& \mathbf{k}_{2}^{*}=\frac{2 \pi}{\operatorname{det}(\tilde{A})}\left(A_{11} \mathbf{e}_{1}+A_{12} \mathbf{e}_{2}\right) .
\end{aligned}
$$

Noting that the $\operatorname{det}(\tilde{A})$ is equal to the area $\mathcal{A}$ of the unit cell, the Hamiltonian (3.13) can then written

$$
\hat{H}=\sum_{n_{1}=-\infty}^{\infty} \sum_{n_{2}=-\infty}^{\infty} \hat{V}_{\mathbf{k}} \hat{T}\left(\frac{h}{e B \mathcal{A}}\left(n_{2} \mathbf{A}_{1}-n_{1} \mathbf{A}_{2}\right)\right)
$$


where $\mathbf{k}=n_{i} \mathbf{a}_{i}$. The elementary transformations associated with this representation of the Hamiltonian, $\hat{T}_{1}=\hat{T}\left(-h \mathbf{A}_{2} / e B \mathcal{A}\right)$ and $\hat{T}_{2}=\hat{T}\left(h \mathbf{A}_{1} / e B \mathcal{A}\right)$ therefore span a lattice which is aligned with the crystal lattice, but scaled by a dimensionless factor

$$
\beta=\frac{h}{e B \mathcal{A}}=\frac{\text { flux quantum }}{\text { flux per unit cell }} .
$$

\section{Extension to irrational magnetic fields}

When the number of flux quanta per unit cell is rational, the spectrum consists of Bloch bands for which Wannier functions have been defined. When the number of flux quanta per unit cell is irrational, there are no Bloch bands and the spectrum is a Cantor set. It is however still possible to define useful sets of generalised Bloch states and corresponding Wannier functions.

The expression giving the Bloch states in terms of the type II Wannier states will be generalised, by writing

$$
\begin{gathered}
|B(\mathbf{k})\rangle=\sum_{\mathbf{R}=n_{1}} \sum_{\mathbf{A}_{1} / N+n_{2} \mathbf{A}_{2}} \exp [-\mathrm{ik} . \mathbf{R}] \sum_{\mu=0}^{|N|-1} \exp \left[2 \pi \mathrm{i} n_{1} \mu / N\right] \\
\times \hat{T}\left(n_{2} \mathbf{A}_{2}\right) \hat{T}\left(n_{1} \mathbf{A}_{1} / N\right) \hat{T}\left(M\left(\mathbf{k} . \mathbf{A}_{1}\right) \mathbf{A}_{2} / \kappa\right)\left|\phi_{\mu}\right\rangle
\end{gathered}
$$

Straightforward application of the composition law (2.1) for magnetic translations to the form (4.1) shows that the generalised Bloch states satisfy a periodicity condition

$$
\left|B\left(\mathbf{k}+\kappa \mathbf{a}_{1} / 2 \pi\right)\right\rangle=\exp \left[\mathrm{i} M\left(\mathbf{k} \cdot \mathbf{A}_{2}\right)\right]|B(\mathbf{k})\rangle
$$

provided that $\exp [2 \pi \mathrm{i} M n / \beta N] \exp [-\mathrm{i} \kappa n / N]=1$ for all integer $n$. The latter condition is used to determine allowed values for the constant $\kappa$ : this quantity must satisfy $\kappa \beta=2 \pi(M+\beta N J)$ with $J$ an integer. Equation (4.2) is a natural generalisation of the periodicity condition $(2.2 \mathrm{c})$. It is desireable to define the generalised Bloch states so that as $\beta \rightarrow p / q$ they converge to the Bloch eigenstates of the rational case with $\beta=p / q$. Setting $J=1$ (and using (1.4)), $\kappa$ aproaches $2 \pi / p$ as $\beta \rightarrow p / q$, which is consistent with (2.2c). The appropriate choice of the constant $\kappa$ defining the dimension of the Brillouin zone is therefore

$$
\kappa \beta=2 \pi(M+\beta N) .
$$

Systematic application of (2.1) shows that the states (4.1) also satisfy other conditions analogous to the standard Bloch states: collecting together the periodicity properties and the equations defining the effect of lattice vector translations, the generalised Bloch states satisfy the relations

$$
\begin{gathered}
\left|B\left(\mathbf{k}+\kappa \mathbf{a}_{1} / 2 \pi\right)\right\rangle=\exp \left[\mathrm{i} M\left(\mathbf{k} \cdot \mathbf{A}_{2}\right)\right]|B(\mathbf{k})\rangle \\
\left|B\left(\mathbf{k}+\mathbf{a}_{2}\right)\right\rangle=|B(\mathbf{k})\rangle \\
\hat{T}\left(\mathbf{A}_{1}\right)|B(\mathbf{k})\rangle=\exp \left[\mathrm{i}\left(\mathbf{k} \cdot \mathbf{A}_{1}\right)\right]\left|B\left(\mathbf{k}-\mathbf{a}_{2} / \beta\right)\right\rangle \\
\hat{T}\left(\mathbf{A}_{2}\right)|B(\mathbf{k})\rangle=\exp \left[\mathrm{i}\left(\mathbf{k} \cdot \mathbf{A}_{2}\right)\right]|B(\mathbf{k})\rangle .
\end{gathered}
$$

Equation (4.1) defined the generalised Bloch states in terms of type II Wannier states. Using the relation between the type I and type II Wannier functions given by (2.4), the 
corresponding relation giving the generalised Bloch states in terms of type I Wannier functions is

$$
\begin{aligned}
& |B(\mathbf{k})\rangle=\sum_{\mathbf{R}=n_{1} \mathbf{A}_{1}+n_{2} \mathbf{A}_{2}} \exp [-\mathrm{ik} \cdot \mathbf{R}] \hat{T}\left(n_{2} \mathbf{A}_{2}\right) \hat{T}\left(n_{1} \mathbf{A}_{1}\right) \\
& \times \hat{T}\left(M\left(\mathbf{k} \cdot \mathbf{A}_{1}\right) \mathbf{A}_{2} / \kappa\right) \sum_{\mu=0}^{|N|-1} \exp \left[-2 \pi \mathrm{i} \mu\left(\mathbf{k} \cdot \mathbf{A}_{1}\right) / \kappa\right]\left|\chi_{\mu}\right\rangle
\end{aligned}
$$

On systematic application of (2.1) and (4.3), it is found that the states (4.5) satisfy the canonical Bloch state relations in the form $(4.4 \mathrm{a}-\mathrm{d})$, for any states $\left|\chi_{\mu}\right\rangle$. The relation between the type I and type II Wannier functions therefore remains valid in the irrational case.

The generalised Bloch states lie in a Brillouin zone spanned by the reciprocal lattice vectors $\kappa \mathbf{a}_{1} / 2 \pi$ and $\mathbf{a}_{2}$, with area $\mathcal{A}_{\mathbf{k}}=\kappa\left|\mathbf{a}_{1} \wedge \mathbf{a}_{2}\right| / 2 \pi$. Applying Born-von Karman boundary conditions, the density of states per unit area associated with the set of generalised Bloch states is $\mathcal{A}_{\mathbf{k}} / 4 \pi^{2}$. The area of the real space unit cell, $\mathcal{A}=\left|\mathbf{A}_{1} \wedge \mathbf{A}_{2}\right|$ is equal to $4 \pi^{2} /\left|\mathbf{a}_{1} \wedge \mathbf{a}_{2}\right|$. The density of generalised Bloch states states per unit area is therefore

$$
\mathcal{N}=\frac{\kappa}{2 \pi \mathcal{A}}
$$

It will now be shown that this density of states is precisely what is required for them to form a complete set of states for a region of the spectrum bounded by two gaps. Středa [15] showed that the density of bulk states per unit area for region of the spectrum bounded by two gaps satisfies is related to the Hall coefficient $\sigma_{x y}$ :

$$
\sigma_{x y}=e \frac{\partial \mathcal{N}}{\partial B}
$$

The Hall coefficient is quantised in units of $e^{2} / h$, and the Chern number $M$ is the quantum number [5]:

$$
\sigma_{x y}=M \frac{e^{2}}{h}
$$

The density of states is clearly correct in the rational case. Using (4.3) with the relation $\beta=h / e B \mathcal{A}$ to differentiate (4.6) with respect to $B$, equation (4.7) reproduces (4.8). This shows that the variation of the density of generalised Bloch states with respect to magnetic field is precisely the same as that of the eigenstates. The generalised Bloch states are therefore a complete set provided that they are not linearly related.

\section{Images of gauge transformations}

The gauge transformations considered are of the form (1.6), in which the Bloch states are multiplied by a factor $\exp [\mathrm{i} \theta(\mathbf{k})]$. The cases of rational and irrational fields will be considered separately.

\subsection{Rational case}

In the rational case $\theta$ satisfies

$$
\theta\left(\mathbf{k}+\mathbf{a}_{1} / p\right)-2 \pi L_{1}=\theta(\mathbf{k})=\theta\left(\mathbf{k}+\mathbf{a}_{2} / p\right)-2 \pi L_{2}
$$


with $L_{1}$ and $L_{2}$ integers, so that the gauge transformation leaves the Bloch states in canonical form. Wannier functions may be defined for the gauge transformed states. These Wannier functions will be different from the original ones, and it is interesting to determine how the transformed Wannier functions may be obtained from the original ones directly.

The calculation will be presented for the special case where

$$
\exp [\mathrm{i} \theta(\mathbf{k})]=\exp \left[\mathrm{ik} \cdot \mathbf{R}^{*}\right]
$$

where

$$
\mathbf{R}^{*}=p\left(L_{1} \mathbf{A}_{1}+L_{2} \mathbf{A}_{2}\right)
$$

is a superlattice vector. More general transformations of the form $\theta(\mathbf{k})=\mathbf{k} . \mathbf{R}^{*}+\epsilon \tilde{\theta}(\mathbf{k})$, with $\tilde{\theta}(\mathbf{k})$ periodic in $k_{1}$ and $k_{2}$, with period $2 \pi / p$, can also be treated for $\epsilon \ll 1$ by Fourier expanding $\tilde{\theta}(\mathbf{k})$. The type I Wannier functions of the gauge transformed Bloch states are

$$
\begin{gathered}
\left|\chi^{\prime}(\mathbf{R})\right\rangle=\frac{p}{4 \pi^{2}} \int_{\mathrm{BZ}} d \mathbf{k} \exp \left[\mathrm{ik} \cdot\left(\mathbf{R}+\mathbf{R}^{*}\right)\right] \hat{T}\left(\frac{-p M}{2 \pi}\left(\mathbf{k} \cdot \mathbf{A}_{1}\right) \mathbf{A}_{2}\right)|B(\mathbf{k})\rangle \\
=\left|\chi\left(\mathbf{R}+\mathbf{R}^{*}\right)\right\rangle .
\end{gathered}
$$

The fundamental type I Wannier functions, $\left|\chi_{\mu}\right\rangle$, are a subset of the full set of Wannier states $|\chi(\mathbf{R})\rangle$, defined by $\left|\chi_{\mu}\right\rangle=\left|\chi\left(p \mu \mathbf{A}_{1}\right)\right\rangle$ : previously the index $\mu$ was restricted to the range $\mu \in\{0, . .,|N|-1\}$ but it is convenient to extend the definition by allowing $\mu$ to take any integer value. The states $|\chi(\mathbf{R})\rangle$ are obtained from the fundamental Wannier functions by the relation [6]

$$
\left|\chi\left(p\left(N n_{1}+\mu\right) \mathbf{A}_{1}+n_{2} \mathbf{A}_{2}\right)\right\rangle=\hat{T}\left(n_{2} \mathbf{A}_{2}\right) \hat{T}\left(n_{1} \mathbf{A}_{1}\right)\left|\chi_{\mu}\right\rangle .
$$

It follows that the extended set of fundamental Wannier states satisfies

$$
\left|\chi_{\mu+N}\right\rangle=\hat{T}\left(\mathbf{A}_{1}\right)\left|\chi_{\mu}\right\rangle
$$

The transformation of the fundamental type I Wannier functions is therefore

$$
\left|\chi_{\mu}^{\prime}\right\rangle=\hat{T}\left(p L_{2} \mathbf{A}_{2}\right)\left|\chi_{\mu+L_{1}}\right\rangle \text {. }
$$

The corresponding transformation of the type II Wannier states is obtained using (2.4) and its inverse relation as follows

$$
\begin{aligned}
& \left|\phi_{\mu}^{\prime}\right\rangle=\frac{1}{N} \sum_{\mu^{\prime}=0}^{|N|-1} \exp \left[-2 \pi \mathrm{i} \mu \mu^{\prime} / N\right] \hat{T}\left(-\mu^{\prime} \mathbf{A}_{1} / N\right)\left|\chi_{\mu^{\prime}}^{\prime}\right\rangle \\
& =\frac{1}{N} \sum_{\mu^{\prime}=0}^{|N|-1} \sum_{\lambda=0}^{|N|-1} \exp \left[2 \pi \mathrm{i}(\lambda-\mu) \mu^{\prime} / N\right] \exp \left[2 \pi \mathrm{i} \lambda L_{1} / N\right] \\
& \times \hat{T}\left(-\mu^{\prime} \mathbf{A}_{1} / N\right) \hat{T}\left(p L_{2} \mathbf{A}_{2}\right) \hat{T}\left(\left(\mu^{\prime}+L_{1}\right) \mathbf{A}_{1} / N\right)\left|\phi_{\lambda}\right\rangle .
\end{aligned}
$$

After combining the translation operators, the summations can be performed: only the term $\lambda=\mu+q L_{2}$ contributes, giving the result

$$
\left|\phi_{\mu}^{\prime}\right\rangle=\exp \left[\frac{2 \pi \mathrm{i}\left(\mu+L_{2} q\right) L_{1}}{N}\right] \hat{T}\left(p L_{2} \mathbf{A}_{2}\right) \hat{T}\left(L_{1} \mathbf{A}_{1} / N\right)\left|\phi_{\mu+q L_{2}}\right\rangle
$$




$$
=\exp \left[\frac{2 \pi \mathrm{i}\left(\mu+\frac{1}{2} q L_{2}\right) L_{1}}{N}\right] \hat{T}\left(L_{1} \mathbf{A}_{1} / N+p L_{2} \mathbf{A}_{2}\right)\left|\phi_{\mu+q L_{2}}\right\rangle .
$$

This expression will be re-cast into a more transparent form in section 8 .

\subsection{Irrational case}

Now consider the case of gauge transformations of the generalised Bloch states defined for irrational fields. In order to define a transformation of the Wannier functions, the gauge transformation must leave the Bloch states in canonical form. If $\beta$ is irrational, equations (4.4b) and (4.4c) imply that a suitable gauge transformation cannot depend upon $k_{2}$. Linear gauge transformations analogous to (5.2) are therefore restricted to being of the form

$$
\left|B^{\prime}(\mathbf{k})\right\rangle=\exp \left[2 \pi \mathrm{i} k_{1} L_{1} / \kappa\right]|B(\mathbf{k})\rangle .
$$

Using (4.1), a Bloch state may be written in terms of the Wannier functions $\left|\phi_{\mu}^{\prime}\right\rangle$ as follows

$$
\begin{gathered}
\left|B^{\prime}(\mathbf{k})\right\rangle=\sum_{n_{1}=-\infty}^{\infty} \sum_{n_{2}=-\infty}^{\infty} \sum_{\mu=0}^{|N|-1} \exp \left[-\mathrm{i} k_{1}\left(n_{1}-L_{1}\right) / N\right] \exp \left[-\mathrm{i} k_{2} n_{2}\right] \exp \left[2 \pi \mathrm{i} \mu\left(n_{1}-L_{1}\right) / N\right] \\
\quad \times \hat{T}\left(n_{2} \mathbf{A}_{2}\right) \hat{T}\left(\left(n_{1}-L_{1}\right) \mathbf{A}_{1} / N\right) \hat{T}\left(M k_{1} \mathbf{A}_{2} / \kappa\right)\left|\phi_{\mu}^{\prime}\right\rangle .
\end{gathered}
$$

If the Wannier functions generating this state are

$$
\left|\phi_{\mu}^{\prime}\right\rangle=\exp \left[2 \pi \mathrm{i} \mu L_{1} / N\right] \hat{T}\left(L_{1} \mathbf{A}_{1} / N\right)\left|\phi_{\mu}\right\rangle
$$

then (using (4.3)) it can be seen that $\left|B^{\prime}(\mathbf{k})\right\rangle$ is related to the original Bloch state by (5.10). This result reduces to a special case of (5.9) in the case where $\beta=p / q$.

\section{Images of translation operators acting upon Wannier states}

This section discusses the states

$$
\hat{T}(\mathbf{r})|B(\mathbf{k})\rangle, \quad \mathbf{r}=\beta\left(\nu_{1} \mathbf{A}_{1}+\nu_{2} \mathbf{A}_{2}\right)
$$

with $\nu_{1}, \nu_{2}$ taking integer values. It will be demonstrated that they are generalised Bloch states of the form (4.1), generated by a set of Wannier functions $\left|\phi_{\mu}^{\prime}\right\rangle, \mu=0, . .,|N|-1$. The transformation giving these Wannier states in terms the states $\left|\phi_{\mu}\right\rangle$ which generate the original Bloch state $|B(\mathbf{k})\rangle$ will be determined. This transformation may be regarded as the image of the operator $T(\mathbf{r})$ acting on the Wannier functions.

The wavevector $\mathbf{k}=\left(k_{1}, k_{2}\right)$ of the state $(6.1)$ is shifted to $\left(k_{1}+\Delta k_{1}, k_{2}\right)$, with $\Delta k_{1}$ to be determined. Commuting the operator $\hat{T}(\mathbf{r})$ to the right using (2.1) gives

$$
\begin{gathered}
\hat{T}(\mathbf{r})|B(\mathbf{k})\rangle=\sum_{n_{1}=-\infty}^{\infty} \sum_{n_{2}=-\infty}^{\infty} \sum_{\mu=0}^{|N|-1} \exp \left[-\mathrm{i}\left(k_{1}+\Delta k_{1}\right) n_{1} / N\right] \exp \left[-\mathrm{i} k_{2} n_{2}\right] \exp \left[2 \pi \mathrm{i} \mu n_{1} / N\right] \\
\times \hat{T}\left(n_{2} \mathbf{A}_{2}\right) \hat{T}\left(n_{1} \mathbf{A}_{1} / N\right) \hat{T}\left(M\left(k_{1}+\Delta k_{1}\right) \mathbf{A}_{2} / \kappa\right) \\
\exp \left[\mathrm{i} \Delta k_{1} n_{1} / N\right] \exp \left[-2 \pi \mathrm{i} \nu_{2} n_{1} / N\right] \exp \left[2 \pi \mathrm{i}\left(k_{1}+\frac{1}{2} \Delta k_{1} M\right) \nu_{1} / \kappa\right] \\
\times \hat{T}\left(\beta \nu_{1} \mathbf{A}_{1}+\left(\beta \nu_{2}-M \Delta k_{1} / \kappa\right) \mathbf{A}_{2}\right)\left|\phi_{\mu}\right\rangle
\end{gathered}
$$


This state is a generalised Bloch state if the product of the final two phase factors containing $n_{1}$ is unity. This occurs if $\Delta k_{1}=2 \pi \nu_{2}$. In this case the argument of the last translation operator simplifies, the multiplier of $\mathbf{A}_{2}$ becoming $2 \pi N \nu_{2} \beta / \kappa$. The state (6.1) is then in the form

$$
\hat{T}(\mathbf{r})|B(\mathbf{k})\rangle=\exp \left[\mathrm{i} \theta\left(\mathbf{k}^{\prime}\right)\right]\left|B^{\prime}\left(\mathbf{k}^{\prime}\right)\right\rangle
$$

where $\left|B^{\prime}(\mathbf{k})\right\rangle$ is a generalised Bloch state with Wannier functions $\left|\phi_{\mu}^{\prime}\right\rangle, \mathbf{k}^{\prime}=\mathbf{k}+\nu_{2} \mathbf{a}_{1}$, and

$$
\theta(\mathbf{k})=\frac{2 \pi k_{1} M \nu_{1}}{\kappa}
$$

The Wannier functions generating $\left|B^{\prime}(\mathbf{k})\right\rangle$ are

$$
\left|\phi_{\mu}^{\prime}\right\rangle=\exp \left[-2 \pi^{2} \mathrm{i} M \nu_{1} \nu_{2} / \kappa\right] \hat{T}\left(\beta \nu_{1} \mathbf{A}_{1}+2 \pi N \beta \nu_{2} \mathbf{A}_{2} / \kappa\right)\left|\phi_{\mu}\right\rangle .
$$

The phase factor in (6.4) represents a gauge transformation of the type (5.10). Using (5.12), we may therfore write

$$
\hat{T}(\mathbf{r})|B(\mathbf{k})\rangle=\left|B^{\prime \prime}\left(\mathbf{k}+\nu_{2} \mathbf{a}_{1}\right)\right\rangle
$$

where the Wannier states generating $|B(\mathbf{k})\rangle$ are

$$
\left|\phi_{\mu}^{\prime \prime}\right\rangle=\exp \left[2 \pi \mathrm{i} M \mu \nu_{1} / N\right] \hat{T}\left(M \nu_{1} \mathbf{A}_{1} / N\right)\left|\phi_{\mu}^{\prime}\right\rangle .
$$

The Wannier functions generating the Bloch states $\left|B^{\prime \prime}\left(\mathbf{k}+\nu_{2} \mathbf{a}_{1}\right)\right\rangle=\hat{T}(\mathbf{r})|B(\mathbf{k})\rangle$ can now be expressed in terms of the original Wannier states:

$$
\left|\phi_{\mu}^{\prime \prime}\right\rangle=\exp \left[\frac{2 \pi \mathrm{i} M \mu \nu_{1}}{N}\right] \hat{T}\left(\frac{\kappa \beta}{2 \pi N} \nu_{1} \mathbf{A}_{1}+\frac{2 \pi N \beta}{\kappa} \nu_{2} \mathbf{A}_{2}\right)\left|\phi_{\mu}\right\rangle .
$$

\section{Dirac brackets of generalised Bloch states}

The objective is to evaluate the matrix element

$$
I\left(\mathbf{k}, \mathbf{k}^{\prime}\right)=\left\langle B^{\prime}\left(\mathbf{k}^{\prime}\right) \mid B(\mathbf{k})\right\rangle
$$

where the $|B(\mathbf{k})\rangle$ and $\left|B^{\prime}(\mathbf{k})\right\rangle$ are different generalised Bloch states for irrational magnetic fields. These Bloch states are generated by different type II Wannier states $\left|\phi_{\mu}\right\rangle$ and $\left|\phi_{\mu}^{\prime}\right\rangle$ respctively, using the expansion (4.1). The resulting expression will later be used to calculate matrix elements of the form $\left\langle B\left(\mathbf{k}^{\prime}\right)|\hat{T}(\mathbf{r})| B(\mathbf{k})\right\rangle$, (where $\mathbf{r}=\nu_{1} \mathbf{a}_{1}+\nu_{2} \mathbf{a}_{2}$ ), and hence matrix elements of the Hamiltonian, by writing $\left|B^{\prime}\left(\mathbf{k}+\nu_{2} \mathbf{a}_{1}\right)\right\rangle=\hat{T}(\mathbf{r})|B(\mathbf{k})\rangle$.

Using (4.1) and (2.1), and writing $k_{i}=\mathbf{k}$. $\mathbf{A}_{i}$, the Dirac bracket is

$$
\begin{gathered}
I=\sum_{n_{1}=-\infty}^{\infty} \sum_{n_{1}^{\prime}=-\infty}^{\infty} \sum_{n_{2}=-\infty}^{\infty} \sum_{n_{2}^{\prime}=-\infty}^{\infty} \sum_{\mu=0}^{|N|-1|N|-1} \sum_{\mu^{\prime}=0} \exp \left[\mathrm{i}\left(k_{2}^{\prime}-k_{2}\right)\left(\frac{n_{2}+n_{2}^{\prime}}{2}\right)\right] \\
\times \exp \left[\mathrm{i}\left(\left(k_{1}^{\prime}-k_{1}\right)+2 \pi\left(\mu-\mu^{\prime}\right)-\frac{2 \pi}{\beta}\left(n_{2}-n_{2}^{\prime}\right)\right)\left(\frac{n_{1}+n_{1}^{\prime}}{2 N}\right)\right] \exp \left[\left(\frac{k_{1}+k_{1}^{\prime}}{2 \kappa}\right)\left(n_{1}^{\prime}-n_{1}\right)\right]
\end{gathered}
$$




$$
\begin{aligned}
& \times \exp \left[\frac{2 \pi \mathrm{i}}{N}\left(\frac{\mu+\mu^{\prime}}{2}\right)\left(n_{1}-n_{1}^{\prime}\right)\right] \exp \left[\mathrm{i}\left(\frac{k_{2}^{\prime}+k_{2}}{2}\right)\left(n_{2}-n_{2}^{\prime}\right)\right] \\
& \times\left\langle\phi_{\mu^{\prime}}^{\prime}\left|\hat{T}\left(\frac{n_{1}-n_{1}^{\prime}}{N} \mathbf{A}_{1}+\left(n_{2}-n_{2}^{\prime}+\frac{M}{\kappa}\left(k_{1}-k_{1}^{\prime}\right)\right) \mathbf{A}_{2}\right)\right| \phi_{\mu}\right\rangle .
\end{aligned}
$$

It is convenient to make changes of variable

$$
\begin{aligned}
& j=n_{1}-n_{1}^{\prime} \quad J=\frac{n_{1}+n_{1}^{\prime}}{2} \\
& l=n_{2}-n_{2}^{\prime} \quad L=\frac{n_{2}+n_{2}^{\prime}}{2} .
\end{aligned}
$$

The summations in (7.2) will then run over integer values of $L$ for even $l$, and over integer plus one half values of $L$ for odd $l$, similarly for $J$ and $j$. These sums are most conveneiently evaluated by decomposing them into four summations:

$$
\begin{gathered}
I=\sum_{j} \sum_{l} \sum_{J} \sum_{L} F(j, l, J, L) \\
=\sum_{n=-\infty}^{\infty} \sum_{n^{\prime}=-\infty}^{\infty} \sum_{m=-\infty}^{\infty} \sum_{m^{\prime}=-\infty}^{\infty}\left[F\left(2 n, 2 m, n^{\prime}, m^{\prime}\right)+F\left(2 n+1, m, n^{\prime}+\frac{1}{2}, m^{\prime}\right)\right. \\
\left.+F\left(2 n, 2 m+1, n^{\prime}, m^{\prime}+\frac{1}{2}\right)+F\left(2 n+1,2 m+1, n^{\prime}+\frac{1}{2}, m^{\prime}+\frac{1}{2}\right)\right] .
\end{gathered}
$$

The function $F(j, l, J, L)$ is of the form

$$
F(j, l, J, L)=\exp \left[\mathrm{i} \alpha_{1}(l) J\right] \exp \left[\mathrm{i} \alpha_{2} L\right] f(j, l)
$$

where

$$
\begin{aligned}
\alpha_{1}(l) & =\frac{1}{N}\left[k_{1}^{\prime}-k_{1}+2 \pi\left(\mu-\mu^{\prime}\right)-\frac{2 \pi}{\beta} l\right] \\
\alpha_{2} & =k_{2}^{\prime}-k_{2}
\end{aligned}
$$

and

$$
\begin{gathered}
f(j, l)=\sum_{\mu=0}^{|N|-1} \sum_{\mu^{\prime}=0}^{|N|-1} \exp \left[-2 \pi \mathrm{i}\left(\frac{k_{1}+k_{1}^{\prime}}{2 \kappa}-\frac{\mu+\mu^{\prime}}{2 N}\right) j\right] \exp \left[\mathrm{i}\left(\frac{k_{2}+k_{2}^{\prime}}{2}\right) l\right] \\
\times\left\langle\phi_{\mu^{\prime}}^{\prime}\left|\hat{T}\left(\frac{j}{N} \mathbf{A}_{1}+\left(l+\frac{M}{\kappa}\left(k_{1}-k_{1}^{\prime}\right)\right) \mathbf{A}_{2}\right)\right| \phi_{\mu}\right\rangle .
\end{gathered}
$$

Using (7.5), it is seen that the sums over $J$ and $L$ are easily evaluated using the Poisson summation formula in the form

$$
\sum_{n=-\infty}^{\infty} \exp (\mathrm{i} \alpha n)=2 \pi \sum_{m=-\infty}^{\infty} \delta(\alpha-2 \pi m)
$$

Using this formula,

$$
I\left(\mathbf{k}^{\prime}, \mathbf{k}\right)=\frac{4 \pi^{2}}{N} \sum_{N_{1}=-\infty}^{\infty} \sum_{N_{2}=-\infty}^{\infty} \sum_{n_{1}=-\infty}^{\infty} \sum_{n_{2}=-\infty}^{\infty}(-1)^{\left(n_{1} N_{1}+n_{2} N_{2}\right)} \delta\left(k_{2}-k_{2}^{\prime}-2 \pi N_{2}\right)
$$




$$
\begin{array}{r}
\times \sum_{\mu=0}^{|N|-1} \sum_{\mu^{\prime}=0}^{|N|-1} \delta\left(k_{1}-k_{1}^{\prime}-2 \pi\left(\mu-\mu^{\prime}\right)+\frac{2 \pi}{\beta} n_{2}-2 \pi N N_{1}\right) \exp \left[\mathrm{i}\left(\frac{k_{2}+k_{2}^{\prime}}{2}\right) n_{2}\right] \\
\times \exp \left[-2 \pi \mathrm{i}\left(\frac{k_{1}+k_{1}^{\prime}}{2 \kappa}-\frac{\mu+\mu^{\prime}}{2}\right) n_{1}\right]\left\langle\phi_{\mu^{\prime}}^{\prime}\left|\hat{T}\left(\frac{n_{1}}{N} \mathbf{A}_{1}+\left(n_{2}+\frac{M}{\kappa}\left(k_{1}-k_{1}^{\prime}\right)\right) \mathbf{A}_{2}\right)\right| \phi_{\mu}\right\rangle .
\end{array}
$$

Writing

$$
\Delta k=2 \pi\left(q-\frac{p}{\beta}\right)
$$

and recalling (4.3), the values of $k_{1}-k_{1}^{\prime}$ for which the matrix element is non-zero may be written in two alternative forms:

$$
k_{1}-k_{1}^{\prime}=l_{1} \Delta k+l_{2} \kappa=2 \pi\left(L_{1}+\frac{1}{\beta} L_{2}\right)
$$

where $l_{1}, l_{2}$ and $L_{1}, L_{2}$ are all integers. The argument of the second delta function in (7.9) can therefore be written in terms of $\Delta k$ and $\kappa$. Noting that

$$
\frac{\partial\left(L_{1}, L_{2}\right)}{\partial\left(l_{1}, l_{2}\right)}=\left|\begin{array}{cc}
q & N \\
-p & M
\end{array}\right|=1
$$

it is seen that the sums over $N_{1}, \mu^{\prime}$ and $n_{2}$ in (7.9) may be replaced by a sum over the indices $l_{1}, l_{2}$ in (7.11). In terms of the new indices $l_{1}, l_{2}$ :

$$
\begin{aligned}
\mu & =\mu^{\prime}+q l_{1}-\lambda N, \quad \lambda=\operatorname{int}\left[\left(\mu^{\prime}+q l_{1}\right) / N\right] \\
N_{1} & =l_{2}+\lambda \\
n_{2} & =p l_{1}-M l_{2} .
\end{aligned}
$$

Also, the argument of the translation operator in (7.9) simplifies, since (using (7.10), (4.3) and (7.11))

$$
\begin{gathered}
n_{2}+\frac{M}{\kappa}\left(k_{1}-k_{1}^{\prime}\right)=\left(p l_{1}-M l_{2}\right)+\frac{M}{\kappa}\left(\Delta k l_{1}+\kappa l_{2}\right) \\
=\left(\frac{M \Delta k}{\kappa}+p\right) l_{1}=\frac{2 \pi}{\kappa} l_{1} .
\end{gathered}
$$

After renaming some of the dummy indices, the Dirac bracket may be written in the form

$$
\begin{gathered}
I\left(\mathbf{k}^{\prime}, \mathbf{k}\right)=\frac{4 \pi^{2}}{N} \sum_{N_{1}=-\infty}^{\infty} \sum_{N_{2}=-\infty}^{\infty} \sum_{n_{1}=-\infty}^{\infty} \sum_{n_{2}=-\infty}^{\infty}(-1)^{N_{2}\left(p n_{1}-M N_{1}\right)+N_{1} n_{2}} \delta\left(k_{2}^{\prime}-k_{2}-2 \pi N_{2}\right) \\
\times \delta\left(k_{1}-k_{1}^{\prime}-N_{1} \kappa-n_{1} \Delta k\right) \exp \left[\mathrm{i}\left(\frac{k_{2}+k_{2}^{\prime}}{2}\right)\left(p n_{1}-M N_{1}\right)\right] \\
\times \sum_{\mu=0}^{|N|-1} \exp \left[-2 \pi \mathrm{i}\left(\frac{k_{1}+k_{1}^{\prime}}{2 \kappa}-\frac{\mu+\mu^{\prime}}{2}\right) n_{2}\right]\left\langle\phi_{\mu}\left|\hat{T}\left(\frac{n_{2}}{N} \mathbf{A}_{1}+\frac{2 \pi}{\kappa} n_{1} \mathbf{A}_{2}\right)\right| \phi_{\mu+q n_{1}+\lambda N}\right\rangle \cdot
\end{gathered}
$$

Using the fact that the type II Wannier functions satisfy $\left|\phi_{\mu+N}\right\rangle=\left|\phi_{\mu}\right\rangle$, the Dirac bracket (7.1) may finally be written in terms of a set of coefficients $I_{n_{1} n_{2}}$ in the form

$$
I\left(\mathbf{k}^{\prime}, \mathbf{k}\right)=\frac{4 \pi^{2}}{N} \sum_{N_{1}=-\infty}^{\infty} \sum_{N_{2}=-\infty}^{\infty} \sum_{n_{1}=-\infty}^{\infty} \sum_{n_{2}=-\infty}^{\infty}(-1)^{N_{1} n_{2}+p N_{2} n_{1}+M N_{1} N_{2}} \delta\left(k_{2}-k_{2}^{\prime}-2 \pi N_{2}\right)
$$




$$
\begin{aligned}
\times \delta\left(k_{1}-k_{1}^{\prime}-\right. & \left.N_{1} \kappa-n_{1} \Delta k\right) \exp \left[\mathrm{i}\left(\frac{k_{2}+k_{2}^{\prime}}{2}\right)\left(p n_{1}-M N_{1}\right)\right] \\
& \times \exp \left[-2 \pi \mathrm{i}\left(\frac{k_{1}+k_{1}^{\prime}}{2 \kappa}\right) n_{2}\right] I_{n_{1} n_{2}} .
\end{aligned}
$$

The coefficients $I_{n_{1} n_{2}}$ are given by

$$
I_{n_{1} n_{2}}=\sum_{\mu=0}^{|N|-1} \exp \left[\frac{2 \pi \mathrm{i}}{N}\left(\mu+\frac{1}{2} q n_{1}\right) n_{2}\right]\left\langle\phi_{\mu}^{\prime}\left|\hat{T}\left(\frac{n_{2}}{N} \mathbf{A}_{1}+\frac{2 \pi}{\kappa} n_{1} \mathbf{A}_{2}\right)\right| \phi_{\mu+q n_{1}}\right\rangle \text {. }
$$

\section{Representations in terms of translation operators}

The expression (7.17) for the coefficients defining the Dirac bracket, and the expression (6.8) for the Wannier function image of a translation operator acting upon a Bloch state, can both be expressed more elegantly by defining extensions of the magnetic translation group.

First I will define a translation operator which acts upon the labels of the type II Wannier states. For integer values of $\lambda_{1}$ and $\lambda_{2}$ they are defined by

$$
\hat{t}\left(n_{1}, n_{2}\right)\left|\phi_{\mu}\right\rangle=\exp \left[\frac{2 \pi \mathrm{i} M}{N}\left(\mu-\frac{1}{2} n_{1}\right) n_{2}\right]\left|\phi_{\mu-n_{1}}\right\rangle .
$$

These operators were originally introduced in [7]. They have an algebra analogous to that of the magnetic translations:

$$
\hat{t}\left(n_{1}, n_{1}\right) \hat{t}\left(n_{1}^{\prime}, n_{2}^{\prime}\right)=\exp \left[-\frac{2 \pi \mathrm{i} M}{N}\left(n_{1} n_{2}^{\prime}-n_{2} n_{1}^{\prime}\right)\right] \hat{t}\left(n_{1}+n_{1}^{\prime}, n_{2}+n_{2}^{\prime}\right) .
$$

Using the definition (8.1), the coefficients $I_{n m}$ given by (7.17) which define the Dirac bracket (7.1) become

$$
I_{n_{1} n_{2}}=(-1)^{p q n_{1} n_{2}} \sum_{\mu=0}^{|N|-1}\left\langle\phi_{\mu}^{\prime}\left|\hat{t}\left(-q n_{1}, q n_{2}\right) \hat{T}\left(\frac{n_{2}}{N} \mathbf{A}_{1}+\frac{2 \pi n_{1}}{\kappa} \mathbf{A}_{2}\right)\right| \phi_{\mu}\right\rangle .
$$

A further simplification can be introduced by using the notation $\mid \Phi)$ to represent the set of $N$ Wannier state vectors $\left\{\left|\phi_{\mu}\right\rangle, \mu=0, . .,|N|-1\right\}$. The object $\left.\mid \Phi\right)$ may be thought of as a state vector in an 'expanded' Hilbert space, with inner product

$$
\left(\Phi^{\prime} \mid \Phi\right)=\sum_{\mu=0}^{|N|-1}\left\langle\phi_{\mu}^{\prime} \mid \phi_{\mu}\right\rangle
$$

Equation (8.3) can now be reduced to a satisfyingly simple form by introducing a generalised magnetic translation operator in the expanded Hilbert space:

$$
\hat{\mathcal{T}}(\mathbf{R})=(-1)^{p q n_{1} n_{2}} \hat{t}\left(-q n_{1}, q n_{2}\right) \hat{T}\left(\frac{n_{2}}{N} \mathbf{A}_{1}+\frac{2 \pi n_{1}}{\kappa} \mathbf{A}_{2}\right), \quad \mathbf{R}=n_{1} \mathbf{A}_{1}+n_{2} \mathbf{A}_{2} .
$$

With this definition

$$
I_{n_{1} n_{2}}=\left(\Phi^{\prime}|\hat{\mathcal{T}}(\mathbf{R})| \Phi\right)
$$


Also, comparing with (5.9), it can be that the gauge transformation exp[ik.R* $\mathbf{R}^{*}$ results in a transformation of the vector of type II Wannier states of the form

$$
\left.\left.\mid \Phi^{\prime}\right)=\hat{\mathcal{T}}\left(\mathbf{R}^{*}\right) \mid \Phi\right) .
$$

The operators $\mathcal{T}(\mathbf{R})$ again have a non-commuting algebra analogous to that of the magnetic translations:

$$
\hat{\mathcal{T}}(\mathbf{R}) \hat{\mathcal{T}}\left(\mathbf{R}^{\prime}\right)=\exp \left[\pi \mathrm{i} \gamma \frac{\left(\mathbf{R} \wedge \mathbf{R}^{\prime}\right)}{\left(\mathbf{A}_{1} \wedge \mathbf{A}_{2}\right)}\right] \hat{\mathcal{T}}\left(\mathbf{R}+\mathbf{R}^{\prime}\right)
$$

where

$$
\gamma=\frac{\Delta k}{\kappa}=\frac{q \beta-p}{M+\beta N}
$$

is the dimensionless magnetic field parameter mentioned in the introduction (equation $(1.5))$.

From (3.16), it is seen that the evaluation of the matrix elements of the Hamiltonian involves calculating the matrix elements $\left\langle B\left(\mathbf{k}^{\prime}\right)|\hat{T}(\mathbf{r})| B(\mathbf{k})\right\rangle$, where $\mathbf{r}=\beta\left(\nu_{1} \mathbf{A}_{1}+\nu_{2} \mathbf{A}_{2}\right)$. The Dirac bracket $\left\langle B^{\prime}\left(\mathbf{k}^{\prime}\right) \mid B(\mathbf{k})\right\rangle$ was obtained in equations (7.16) and (7.17) in terms of a set of coefficients $I_{n_{1} n_{2}}$. The calculation of section 6 shows that the operator $\hat{T}(\mathbf{r})$ acting on a Bloch state creates a new canonical Bloch state with $\mathbf{k}$ shifted to $\mathbf{k}+\nu_{2} \mathbf{a}_{1}$. It is natural to expect that the Dirac bracket $\left\langle B^{\prime}\left(\mathbf{k}^{\prime}\right) \mid B\left(\mathbf{k}+\nu_{2} \mathbf{a}_{1}\right)\right\rangle$ may be expressed in the form (7.16), with the coefficients $I_{n_{1} n_{2}}$ replaced by $I_{\Phi^{\prime} \Phi}\left(n_{1}, n_{2}, \nu_{2}\right)$ (note that $I_{n_{1} n_{2}}=I_{\Phi^{\prime} \Phi}\left(n_{1}, n_{2}, 0\right)$ ). Noting that $p \kappa+M \Delta k=2 \pi$, and that $k_{1}$ is replaced by $k_{1}+2 \pi \nu_{2}$ in (7.16), it is found that

$$
\begin{gathered}
\left\langle B^{\prime}\left(\mathbf{k}^{\prime}\right) \mid B\left(\mathbf{k}+\nu_{2} \mathbf{a}_{1}\right)\right\rangle=\sum_{N_{1}=-\infty}^{\infty} \sum_{N_{2}=-\infty}^{\infty} \sum_{n_{1}=-\infty}^{\infty} \sum_{n_{2}=-\infty}^{\infty}(-1)^{N_{1} n_{2}+p N_{2} n_{1}+M N_{1} N_{2}} \\
\times \delta\left(k_{2}-k_{2}^{\prime}-2 \pi N_{2}\right) \delta\left(k_{1}-k_{1}^{\prime}-N_{1} \kappa-n_{1} \Delta k\right) \exp \left[\mathrm{i}\left(\frac{k_{2}+k_{2}^{\prime}}{2}\right)\left(p n_{1}-M N_{1}\right)\right] \\
\times \exp \left[-2 \pi \mathrm{i}\left(\frac{k_{1}+k_{1}^{\prime}}{2 \kappa}\right) n_{2}\right] I_{\Phi^{\prime} \Phi}\left(n_{1}, n_{2}, \nu_{2}\right)
\end{gathered}
$$

where

$$
I_{\Phi^{\prime} \Phi}\left(n_{1}, n_{2}, \nu_{2}\right)=\exp \left[\mathrm{i} \pi(p+2 \pi / \kappa) n_{2} \nu_{2}\right] I_{n_{1}+M \nu_{2}, n_{2}}
$$

Now consider the evaluation of the matrix element $\left\langle B^{\prime}\left(\mathbf{k}^{\prime}\right)|\hat{T}(\mathbf{r})| B(\mathbf{k})\right\rangle$. This may be written in the form (8.10), with the Wannier state $\mid \Phi)$ replaced by the state $\left.\mid \Phi^{\prime \prime}\right)$ given by (6.8). Combining (6.8) and (8.11), the coefficients may be written in the form (8.10) with coefficients

$$
\begin{gathered}
I_{\Phi^{\prime} \Phi^{\prime \prime}}\left(n_{1}, n_{2}, \nu_{1}, \nu_{2}\right)=\exp \left[\mathrm{i} \pi(p+2 \pi / \kappa) n_{2} \nu_{2}\right] \sum_{\mu=0}^{|N|-1} \exp \left[\frac{2 \pi \mathrm{i} M \mu \nu_{1}}{N}\right] \\
\times\left\langle\phi_{\mu}^{\prime}\left|\hat{\mathcal{T}}\left(\mathbf{R}+M \nu_{2} \mathbf{A}_{1}\right) \hat{T}\left(\frac{\kappa \beta}{2 \pi N} \nu_{1} \mathbf{A}_{1}+\frac{2 \pi N \beta}{\kappa} \nu_{2} \mathbf{A}_{2}\right)\right| \phi_{\mu}\right\rangle .
\end{gathered}
$$

This coefficient may be expressed in the form

$$
I_{\Phi^{\prime} \Phi^{\prime \prime}}\left(n_{1}, n_{2}, \nu_{1}, \nu_{2}\right)=\left(\Phi^{\prime}|\hat{\mathcal{T}}(\mathbf{R}) \hat{\tau}(\mathbf{r})| \Phi\right)
$$


where

$$
\hat{\tau}(\mathbf{r})=\hat{t}\left(-\nu_{2}, \nu_{1}\right) \hat{T}\left(\frac{\kappa \beta}{2 \pi N} \nu_{1} \mathbf{A}_{1}+\beta \nu_{2} \mathbf{A}_{2}\right) .
$$

The operators $\tau(\mathbf{r})$ commute with the $\hat{\mathcal{T}}(\mathbf{R})$ operators:

$$
[\hat{\tau}(\mathbf{r}), \hat{\mathcal{T}}(\mathbf{R})]=0
$$

for all lattice vectors $\mathbf{R}$ and $\mathbf{r} / \beta$.

\section{The generalised Peierls effective Hamiltonian}

\subsection{A one dimensional effective Hamiltonian}

The motivation is to obtain an effective Hamiltonian, having a spectrum which is the same as a subset of the spectrum of the original Hamiltonian. The effective Hamiltonian is easier to analyse because the number of degrees of freedom has been reduced. The approach is analogous to that used in earlier work on the phase space lattice Hamiltonian $[7,14]$. The Hamiltonian will be reduced to a block diagonal form, and matrix elements of the Hamiltonian within one block will be compared with matrix elements of the effective Hamiltonian. If the basis states are in one to one correspondence and the matrix elements are equal, then the spectrum of the effective Hamiltonian is the same as that of the block of the full Hamiltonian.

In the case under consideration, matrix elements of the Hamiltonian will be evaluated in the basis formed by a set of generalised Bloch states $\left|B^{\prime}(\mathbf{k})\right\rangle$. They are compared with matrix elements of an effective Hamiltonian $\hat{H}_{\text {proj }}$ in a suitable basis with elements $\left|\bar{\xi}\left(x, k_{2}\right)\right\rangle$, and the coefficients defining $\hat{H}_{\text {proj }}$ are chosen such that the non-zero matrix elements of $\hat{H}_{\text {proj }}$ correspond with those of $\hat{H}$, in that

$$
\left\langle B\left(\mathbf{k}^{\prime}\right)|\hat{H}| B(\mathbf{k})\right\rangle=\frac{4 \pi^{2}}{N \kappa}\left\langle\bar{\xi}\left(x^{\prime}, k_{2}\right)\left|\hat{H}_{\text {proj }}\right| \bar{\xi}\left(x, k_{2}\right)\right\rangle \delta\left(k_{2}-k_{2}^{\prime}\right) .
$$

This equality holds when $k_{2}=k_{2}^{\prime}$, and where the states $\left|\bar{\xi}\left(x, k_{2}\right)\right\rangle$ are labelled by a continuous variable $x=k_{1} / \kappa$.

It will be assumed that in the case where the dimensionless magnetic field $\beta$ takes the rational value $p / q$, there is a non-degenerate band. It will also be assumed that the gap separating this band from the rest of the spectrum does not close when $\beta$ is perturbed away from the rational value $p / q$. The effective Hamiltonian is constructed to reproduce that part of the full spectrum which evolves out this band when $\beta$ is perturbed from the rational value. The type II Wannier functions $\left|\phi_{\mu}\right\rangle$ for this band are determined, and used to generate a set of generalised Bloch states using (4.1). A projection operator $\hat{P}=f(\hat{H})$ is applied to these states, where the function $f(E)$ is unity where $E$ lies inside the band, and zero throughout the rest of the spectrum. The states resulting from applying this projection

$$
\left|B^{\prime}(\mathbf{k})\right\rangle=\hat{P}|B(\mathbf{k})\rangle
$$

are orthogonal to all eigenstates outside the band, and therefore represent the Hamiltonian in block diagonal form. The projection operator may be written in the form

$$
\hat{P}=\int_{-\infty}^{\infty} d t \tilde{f}(t) \exp [\dot{\mathrm{i}} \hat{H} t]
$$


where $\tilde{f}(t)$ is a Fourier transform of $f(E)$. The stipulation that the spectrum has a gap on either side of the band ensures that $f(E)$ can have arbitrarily many continuous derivatives, implying that this integral is nicely behaved.

The projected Bloch generalised Bloch states are sufficiently numerous to form a complete but not overcomplete set for the band, and may be assumed to be complete provided the matrix element $\left\langle B^{\prime}\left(\mathbf{k}^{\prime}\right) \mid B^{\prime}(\mathbf{k})\right\rangle$ is sufficiently small when $\mathbf{k} \neq \mathbf{k}^{\prime}$. This criterion can be tested and verified using the results of sections 7 and 8 . Because the states are not orthonormal, a normalisation operator must also be calculated, such that

$$
\left\langle B^{\prime}\left(\mathbf{k}^{\prime}\right) \mid B^{\prime}(\mathbf{k})\right\rangle=\frac{4 \pi^{2}}{N \kappa}\left\langle\bar{\xi}\left(x^{\prime}, k_{2}\right)\left|\hat{N}_{\text {proj }}\right| \bar{\xi}\left(x, k_{2}\right)\right\rangle \delta\left(k_{2}-k_{2}^{\prime}\right) \text {. }
$$

The subset of the spectrum of the full Hamiltonian which lies in the projected band can be determined exactly by solving the eigenvalue problem $\left[\hat{H}_{\text {proj }}-E \hat{N}_{\text {proj }}\right]|\psi\rangle=0$, or alternatively by calculating the spectrum of the effective Hamiltonian operator

$$
\hat{H}_{\text {eff }}=\hat{N}_{\text {proj }}^{-1 / 2} \hat{H}_{\text {proj }} \hat{N}_{\text {proj }}^{-1 / 2}
$$

Consider the matrix elements of the Hamiltonian, expressed in the form (3.16), in the basis formed by the generalised Bloch states. The wavevectors $\mathbf{k}$ and $\mathbf{k}^{\prime}$ can both be restricted to the first Brillouin zone, i.e. $k_{1}, k_{1}^{\prime} \in[0, \kappa)$ and $k_{2}, k_{2}^{\prime} \in[0,2 \pi)$, because these states form a complete set. Alternatively, states in an extended Brillouin zone can be used, since they only differ by a phase factor from the states within the first Brillouin zone. States with $k_{1}$ differing by multiples of $\kappa$ are identical (apart from a phase factor). Similiarly, states with $k_{2}$ differing by multiples of $2 \pi$ are identical. When writing matrix elements of the Hamiltonian in a complete set of states, the summations over $N_{1}$ and $N_{2}$ in (7.16) can therefore be dropped:

$$
\begin{aligned}
& \left\langle B\left(\mathbf{k}^{\prime}\right)|\hat{H}| B(\mathbf{k})\right\rangle=\frac{4 \pi^{2}}{N} \delta\left(k_{2}-k_{2}^{\prime}\right) \sum_{n_{1}=-\infty}^{\infty} \delta\left(k_{1}-k_{1}^{\prime}-n_{1} \Delta k\right) \\
& \quad \times \exp \left[\mathrm{i} p k_{2} n_{1}\right] \sum_{n_{2}=-\infty}^{\infty} \exp \left[-2 \pi \mathrm{i}\left(\frac{k_{1}+k_{1}^{\prime}}{2 \kappa}\right) n_{2}\right] H_{n_{1} n_{2}}^{\prime} .
\end{aligned}
$$

In the case where $\beta$ is rational, $n \kappa+m \Delta k=0$ for some choice of $n$ and $m$. In particular, $\gamma=\Delta k / \kappa$ is also a rational number, $\gamma=p^{\prime} / q^{\prime}$, so that this relationship is satisfied when $\mathrm{n}$ is a multiple of $q^{\prime}$. In this case, only $q^{\prime}$ distinct states are coupled, and the Hamiltonian is represented by a $q^{\prime} \times q^{\prime}$ matrix with parameters $k_{2} \in[0,2 \pi)$ and $k_{1} \in\left[0, \kappa / q^{\prime}\right)$. In the general case there is no finite dimensional representation.

Now compare the matrix elements (9.6) with matrix elements of an effective Hamiltonian of the form

$$
\hat{H}_{\text {proj }}=H_{\text {proj }}(\hat{\mathbf{K}})=\sum_{\mathbf{R}} H^{\prime}(\mathbf{R}) \exp [\mathrm{i} \hat{\mathbf{K}} \cdot \mathbf{R}] \equiv \sum_{\mathbf{R}} H^{\prime}(\mathbf{R}) \hat{T}^{\prime}(\mathbf{R})
$$

where sum runs over all of the lattice vectors $\mathbf{R}=n_{1} \mathbf{A}_{1}+n_{2} \mathbf{A}_{2}$, and where the following relations hold:

$$
\begin{gathered}
\hat{\mathbf{K}}=\frac{1}{2 \pi}\left(\mathbf{a}_{1} \hat{g}_{1}+\mathbf{a}_{2} \hat{g}_{2}\right) \\
{\left[\hat{g}_{1}, \hat{g}_{2}\right]=2 \pi \mathrm{i} \gamma}
\end{gathered}
$$


(here the $\mathbf{a}_{i}$ are reciprocal lattice vectors, satisfying $\mathbf{a}_{i} \cdot \mathbf{A}_{j}=2 \pi \delta_{i j}$ ). The operators $\hat{g}_{1}$ and $\hat{g}_{2}$ have a commutator which is analogous to the usual position and momentum operators. Eigenstates of $\hat{g}_{2}$ will be introduced, with eigenvalue $x: \hat{g}_{2}|\xi(x)\rangle=x|\xi(x)\rangle$. Evaluating the matrix elements of (9.7) in this basis leads to matrix elements which are very similar in structure to (9.6), if the coefficients $H^{\prime}(\mathbf{R})$ in (9.7) are identified with the coefficients $H_{n_{1} n_{2}}^{\prime}$ in (9.6). The correspondence becomes even closer if the states $|\xi(x)\rangle$ are 'gaugetransformed' as follows:

$$
\left|\bar{\xi}\left(x, k_{2}\right)\right\rangle=\exp \left[\mathrm{i}\left(\frac{p k_{2}}{2 \pi \gamma}\right) x\right]|\xi(x)\rangle
$$

The matrix elements are then

$$
\begin{gathered}
\left\langle\bar{\xi}\left(x^{\prime}, k_{2}\right)\left|\hat{H}_{\text {proj }}\right| \bar{\xi}\left(x, k_{2}\right)\right\rangle=\sum_{n_{1}=-\infty}^{\infty} \sum_{n_{2}=-\infty}^{\infty} H^{\prime}(\mathbf{R}) \exp \left[\mathrm{i}\left(x+x^{\prime}\right) n_{2} / 2\right] \\
\times \exp \left[\mathrm{i} p n_{1} k_{2}\right] \delta\left(x-x^{\prime}-2 \pi \gamma n_{1}\right) .
\end{gathered}
$$

Identifying $x=k_{1} / \kappa$ and $\gamma=\Delta k / \kappa$, these matrix elements of $\hat{H}_{\text {proj }}$ are identical to the elements (9.6) for all values of $k_{2}$. The spectrum of (9.7) is therefore identical to that of (9.6) when $\gamma=\Delta k / \kappa$ and $H^{\prime}(\mathbf{R})=H_{n_{1} n_{2}}^{\prime}$.

\section{Coefficients of the effective Hamiltonian}

It remains to determine the coefficients $H^{\prime}(\mathbf{R})=H_{n_{1} n_{2}}^{\prime}$ in (9.6). These are obtained using (7.16) and the notational devices introduced in section 8. The Hamiltonian is given by (3.16), and takes the form of a sum of magnetic translations of the form $\hat{T}(\mathbf{r})$, where $\mathbf{r} / \beta$ are lattice vectors. The action of the Hamiltonian (3.16) upon a Bloch state $|B(\mathbf{k})\rangle$ may be represented in terms of the action of an image Hamiltonian upon the Wannier states that generate the Bloch states. The matrix elements of the Hamiltonian, $\left\langle B\left(\mathbf{k}^{\prime}\right)|\hat{H}| B(\mathbf{k})\right\rangle$, are of the form (8.10), with the coefficients $I_{\Phi^{\prime} \Phi}\left(n_{1}, n_{2}, \nu_{2}\right)$ replaced by coefficients $H_{n_{1} n_{2}}^{\prime}=$ $H^{\prime}(\mathbf{R})$ characterising the Hamiltonian. These are given by an expression analogous to (8.13):

$$
H^{\prime}(\mathbf{R})=(\Phi|\hat{\mathcal{T}}(\mathbf{R}) \hat{\mathcal{H}}| \Phi)
$$

The operators $\hat{V}_{\mathbf{k}}$ in (3.16) commute with the magnetic translations, and therefore commute with $\hat{\tau}(\mathbf{r})$ and $\hat{\mathcal{T}}(\mathbf{R})$. Using (8.13) and (3.16), it is seen that the operator $\hat{\mathcal{H}}$, which is the image of the Hamiltonian in the Wannier function Hilbert space, is

$$
\hat{\mathcal{H}}=\sum_{\mathbf{k}} \hat{V}_{\mathbf{k}} \hat{\tau}(\mathbf{r}(\mathbf{k}))
$$

where $\mathbf{r}(\mathbf{k})=\beta\left(n_{2} \mathbf{A}_{1}-n_{1} \mathbf{A}_{2}\right)$ corresponds to the reciprocal lattice vector $\mathbf{k}=n_{1} \mathbf{a}_{1}+n_{2} \mathbf{a}_{2}$. The image $\hat{\mathcal{H}}$ of Hamiltonian in the space of the Wannier states commutes with the image of the lattice translation operators:

$$
[\hat{\mathcal{H}}, \hat{\mathcal{T}}(\mathbf{R})]=0
$$

A similar representation exists for the projection operator $\hat{P}=f(\hat{H})$ : this has an image in the form of an operator $\hat{\mathcal{P}}$ acting upon the Wannier states. Also, the operator $\hat{\mathcal{H}}_{\text {proj }}=\hat{\mathcal{P}} \hat{\mathcal{H}} \hat{\mathcal{P}}$ which is the image of the projected Hamiltonian $\hat{H}_{\text {proj }}$ acting on the Wannier 
functions may also be expressed in a form analogous to (9.13). The effective Hamiltonian can also be represented by an operator $\hat{\mathcal{H}}_{\text {eff }}=\hat{\mathcal{P}}^{-1 / 2} \hat{\mathcal{H}} \hat{\mathcal{P}}^{-1 / 2}$ acting on the Wannier states.

The formulae discussed above can be used to calculate the Fourier coefficients of the effective Hamiltonian using (9.12). Methods for calculating these coefficients as an expansion in $\beta-p / q$ are discussed in [14] for the case of the phase space lattice Hamiltonian, and these techniques may be adapted to the present problem. In order to establish the validity of the Peierls formula, it is necessary only to establish the limit of the coefficients $H^{\prime}(\mathbf{R})$ in the limit $\beta \rightarrow p / q$. These coefficients are identified by noting that, upon setting $\beta=p / q$ the Bloch states become eigenstates, so that $\left\langle B\left(\mathbf{k}^{\prime}\right)|\hat{H}| B(\mathbf{k})\right\rangle=\mathcal{E}(\mathbf{k}) \delta\left(\mathbf{k}-\mathbf{k}^{\prime}\right)$. The corresponding expression (9.11) for the matrix elements of the effective Hamiltonian reduces to

$$
\left\langle\bar{\xi}\left(x^{\prime}, k_{2}\right)\left|\hat{H}_{\mathrm{eff}}\right| \bar{\xi}\left(x, k_{2}\right)\right\rangle=\sum_{n_{1}=-\infty}^{\infty} \sum_{n_{2}=-\infty}^{\infty} H^{\prime}(\mathbf{R}) \delta\left(x-x^{\prime}\right) \exp \left[\mathrm{i} x n_{2}\right] \exp \left[-\mathrm{i} p n_{1} k_{2}\right] .
$$

In the limit $\beta \rightarrow p / q$, the coefficients $H^{\prime}(\mathbf{R})$ of the effective Hamiltonian are therefore the Fourier coefficients of the dispersion relation. The effective Hamiltonian (9.7) is therefore of the 'Peierls substitution' form, (1.1).

\section{Acknowledgements}

This work was supported by the EPSRC, research grant reference GR/L02302. 


\section{References}

[1] G. H. Wannier, Phys. Rev., 52, 191, (1937).

[2] M. Ya. Azbel, Zh. eksp. teor. Fiz., 46, 929, (1964). (transl. Sov. Phys. JETP, 19, 634-45, (1964)).

[3] D. R. Hofstadter, Phys. Rev., B14, 2239-49, (1976).

[4] D. J. Thouless, J. Phys., C17, 325-8, (1984).

[5] D. J. Thouless, M. Kohmoto, M. P. Nightingale, and M. den Nijs, Phys. Rev. Lett., 49, 405-8, (1982).

[6] M. Wilkinson, J. Phys.: Condensed Matter, 10, 7407, (1998).

[7] M. Wilkinson, J. Phys., A27, 8123-48, (1994).

[8] M. Wilkinson and R. J. Kay, J. Phys., A30, 5551, (1997).

[9] R. E. Peierls, Z. Phys., 80, 763-91, (1933).

[10] P. G. Harper, Proc. Phys. Soc., A68, 879-92, (1955).

[11] R. Brown, Phys. Rev., A133, 1038, (1964).

[12] J. Zak, Phys. Rev., A134, 1602, (1964).

[13] M. C. Chang and Q. Niu, Phys. Rev., B53, 7010, (1996).

[14] R. J. Kay and M. Wilkinson, J. Phys., A29, 1515, (1996).

[15] P. Středa, J. Phys., C15, L717-27, (1982). 